\title{
Traduire
}

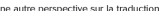

Revue française de la traduction

$244 \mid 2021$

Des jeux et des mots

\section{Traduire le jeu vidéo : rêves et réalités. Entretien autour de l'expérience de cinq traductrices}

Sandra Mouton

\section{(2) OpenEdition \\ Journals}

Édition électronique

URL : https://journals.openedition.org/traduire/2308

DOI : $10.4000 /$ traduire.2308

ISSN : 2272-9992

Éditeur

Société française des traducteurs

Édition imprimée

Date de publication : 15 juin 2021

Pagination : 51-64

ISSN : 0395-773X

\section{Référence électronique}

Sandra Mouton, «Traduire le jeu vidéo : rêves et réalités. Entretien autour de l'expérience de cinq traductrices », Traduire [En ligne], 244 | 2021, mis en ligne le 15 juin 2021, consulté le 01 juillet 2021 URL : http://journals.openedition.org/traduire/2308; DOI : https://doi.org/10.4000/traduire.2308 


\section{Traduire le jeu vidéo: rêves et réalités}

\section{Entretien autour de l'expérience de cinq traductrices}

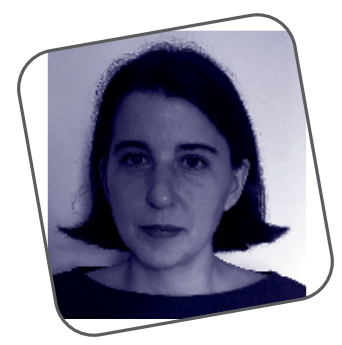

\section{Sandra Mouton}

La traduction de jeux vidéo suscite quantité de discours, de la presse affichant des chiffres de recettes du secteur à douze mille zéros aux commentaires amusés ou envieux des extérieurs au sérail qui répondent d'un «ça a l'air fun comme boulot!» quand on leur parle de la traduction du moment. Si les publications et sites spécialisés dans le vidéoludique consacrent à l'occasion un dossier ou un billet au métier de la localisation et à ses problématiques, si les réseaux sociaux fourmillent de discussions étrillant le dernier fail de traduction ou les conditions misérables faites à certains professionnels, les éléments concrets sur le travail effectivement réalisé par celles et ceux qui font passer à un jeu les frontières de sa langue originale brillent par leur absence.

II se trouve que ce domaine méconnu est un de ceux dans lesquels je traduis. Au fil des ans et des jeux, j'ai constaté que ce travail était différent d'autres types de traduction et, en lui-même, très varié. J'ai donc eu envie de confronter mon 
expérience à celle de consœurs qui, elles aussi, travaillent sur le jeu vidéo.

Conversation entre Sandra Mouton (anglais/russe/latin > français), Elisabeth Monrozier (anglais/russe > français), Jessica West (russe/allemand $>$ anglais), Sasha Barral (français > anglais) et Xiaochun Zhang (anglais > chinois).

\section{Des textes-source variés}

La traduction de jeux vidéo recouvre tout un éventail de textes pouvant se situer dans le jeu, à l'extérieur du jeu ou "parler sur»le jeu. Parmi les contenus in-game', on trouve l'interface utilisateur (UI, de l'anglais user interface), les noms d'objets, d'équipements, de compétences, etc., les parties narratives et les dialogues, écrits ou audio (avec doublage ou sous-titrage). Comme pour n'importe quel produit, le jeu rassemble aussi autour de lui des contenus marketing, descriptions et offres promotionnelles et des textes juridiques (contrats de licence, politique de confidentialité et de protection des données...). Enfin, il existe des contenus qui parlent du jeu de façon technique, par exemple les blogs de développeurs et les notes de version et de patch.

Quel que soit le type de contenu, nous sommes d'accord toutes les cinq: le format du texte-source est invariablement un tableur de dizaines de milliers de lignes (à raison d'une string ${ }^{2}$ par ligne) ou son équivalent dans le logiciel de TAO ou la plateforme de gestion de contenu choisis par le client.

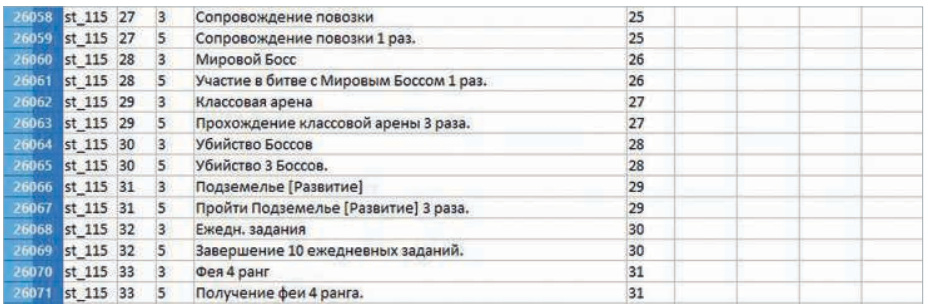

1. Internes au jeu.

2. Unité de texte. 


\section{Quid du contexte?}

Le problème saute aux yeux: où donc est le contexte? Plus, peut-être, que pour tout autre texte à traduire, le contenu ingame exige de savoir qui parle à qui, quand, où et pour dire quoi. Les configurations rencontrées par les traductrices sur ce point crucial varient énormément.

Sandra Mouton: On a parfois la chance de tomber sur des développeurs qui ont adopté un système de string $1 D^{3}$ cohérent et transparent et intégré des explications au fichiersource. Si le projet est bien organisé, un système sera prévu pour poser des questions aux développeurs ou à l'éditeur et voir des captures d'écran.

\begin{tabular}{|l|l|l|l|}
\hline String ID & Source & Translation & Note \\
\hline UI_GENERAL_IDENTIFY & Identify & & $\begin{array}{l}\text { Context: Rare items have } \\
\text { stats hidden unless you use } \\
\text { an 'identify scroll' on them to } \\
\text { discover what the stats are }\end{array}$ \\
\hline
\end{tabular}

Elisabeth Monrozier: C'est assez perturbant d'avoir des strings sans aucun contexte, sans même savoir si ce sont des phrases qui s'afficheront à la suite les unes des autres à l'écran. On traduit vraiment à l'aveuglette.

Xiaochun Zhang: Je reçois souvent des documents de référence, comme une présentation de l'histoire, les demandes du client, des images des personnages principaux, etc. De plus en plus, j'ai accès à des démos du jeu et parfois, je peux même jover à une version de travail.

Jessica West: Je pense que, dans le monde anglophone, le fait de ne pas toujours avoir accès au jeu lui-même ou de ne pas pouvoir communiquer avec ses créateurs vient d'un sentiment d'insécurité autour de la propriété intellectuelle et de la peur que le contenu du jeu fuite avant la sortie.

Sasha Barral: Les très gros studios envoient de la documentation, ce dont je suis toujours contente. Les meilleurs fournissent aussi un guide de style, ce qui est génial. En général, on reçoit aussi des descriptions des personnages, un résumé de l'histoire, parfois un glossaire du jeu.

3. Série de caractères identifiant chaque string dans l'économie et le système informatique d'un jeu donné. 
SM: Ça peut être à double tranchant, d'avoir un développeur qui donne accès au jeu, s'il n'y a rien d'autre. On n'a pas forcément le temps d'aller farfouiller. Une fois, pour déterminer si скала désignait une falaise ou un rocher, j'ai dû créer un personnage, armer un bateau et naviguer sous la mitraille jusqu'au lieu dont je devais traduire le nom. J'ai été coulée six fois avant d'y arriver! C'était plutôt rigolo, mais heureusement que le reste du contexte était clair: le développeur, qui travaillait seul, était submergé et n'avait pas du tout le temps de répondre.

JW: On est parfois obligé de partir à la pêche aux informations. Pour traduire un blog de devs rempli de réflexions sur la physique de l'animation de l'eau, j'ai passé des heures à regarder des vidéos YouTube pour essayer d'y comprendre quelque chose.

\section{Oh my god, it's alive!}

$X Z$ : Dans le monde interactif du jeu, chaque joueur est à la fois spectateur et participant. Les traducteurs auront donc affaire à des textes fragmentés et non linéaires soumis à toutes sortes de restrictions techniques et devront assurer la jouabilité du jeu.

En d'autres termes, foin de la quête de correction traductologique ou de beauté pure, il faut que la mécanique du jeu puisse se mettre en branle. Pour tous les éléments que le joueur va utiliser, la précision est vitale. Armes, créatures, véhicules, objets, sorts, etc. Pas question de fusionner des termes différents ou de varier la traduction d'un terme technique ou spécifique.

EM: Comme le russe fait beaucoup d'emprunts aux langues occidentales, on se retrouve avec des doublons parfois difficiles à traduire différemment en français. Je me souviens d'une liste de noms de créatures où on retrouvait великан et гигант. Les deux mots n'ont pas réellement un sens différent, mais il s'agissait de deux êtres distincts dans le jeu. Impossible de les appeler tous les deux «géant» et bien sûr «titan» était déjà pris.

Le traitement des nombres et autres variables (qui peuvent être des couleurs, des créatures, etc.) est très important. 
Les chaînes de caractères des variables et des $\operatorname{tags}^{4}$ ne doivent surtout pas être modifiées au risque de saboter cette partie du jeu et la phrase doit fonctionner pour les différentes valeurs possibles des variables.

\begin{tabular}{|l|l|}
\hline Source Language Text & French Text (Translation) \\
\hline & \\
\hline Силы тьмы увеличивают атаку на & Les forces occultes augmentent \\
[AbilityParam] \{buff_param_0\} & ['Attaque de [AbilityParam] \\
[CloseParam]. Длительность: & \{buff_param_0\}[CloseParam] pendant \\
[AbilityParam]\{buff_duration\} & [AbilityParam]\{buff_duration\} \\
[CloseParam] ход(a). & [CloseParam] tour(s). \\
\hline
\end{tabular}

La situation se présente très différemment selon les langues.

$\mathbf{X Z}$ : Le chinois, d'une manière générale, pose peu problème pour les questions de variables et de nombres.

SM: C'est un problème en français avec les marques de pluriel et de genre, par exemple des mots en -eux et en-al, où on ne peut pas s'en tirer avec un s et/ou un e entre parenthèses.

EM: La traduction des $\mathrm{PV}$ et $X \mathrm{P}^{5}$ peut poser problème en russe à cause des déclinaisons: pour des questions de place, la fin des mots est parfois abrégée, mais quand il manque la terminaison, difficile de rétablir le sens.

D'autres aspects, plus subtils, influent sur l'expérience de jeu. JW: Un nombre de caractères relativement faible à l'écran diminue la charge cognitive du joueur et augmente son immersion. Le russe a une tradition argotique très riche (plus qu'en anglais, je trouve) et c'est parfois difficile de trouver une solution qui n'aplatisse pas le texte, pour préserver l'immersion.

\section{Un monde à part}

SB: Le registre de langue est volontiers familier. II peut y avoir des jurons, des insultes, de l'argot. II y a aussi un jargon spécifique.

XZ: Les traducteurs de jeux doivent connaître l'argot internet chinois. Sous l'influence de l'anglais des forums, il contient

4. Ou balises.

5. Les sacro-saints points de vie et points d'expérience. 
notamment des acronymes d'expressions en pinyin ${ }^{6}$, par exemple BT pour biàn tài (变态, «pervers, anormal»). On ne peut donc pas utiliser l'acronyme BT dans un autre sens.

JW:I existe tout un ensemble de normes, une terminologie, exactement comme pour la traduction juridique ou financière. Par exemple, les normes sur l'utilisation des majuscules. J'ai travaillé sur des jeux où les noms communs Town, Castle, Troops, Equipment avaient toujours la majuscule, ce qui est contre-intuitif.

EM: On trouve un joyeux mélange de mythologies, grecque, scandinave, hébraïque, etc., qui crée une mythologie spécifique, une culture propre au jeu vidéo, pas très différenciée d'un pays à l'autre, je suppose.

Chaque jeu possède aussi son propre univers et son vocabulaire. Pour les jeux à plusieurs épisodes et pour les extensions, si on prend la franchise en route, on est tenu par les choix de localisation faits par d'autres auparavant.

EM: En l'absence de mémoire de traduction, ou si elle est incohérente, s'il n'y a pas de base terminologique, il est difficile de connaître les termes déjà utilisés dans le jeu ou dans des communications précédentes.

SM: Même si on n'approuve pas les choix passés, ils sont gravés dans le marbre. Dans les listes de créatures monstrueuses d'un jeu de rôle, je me suis aperçue qu'une plante carnivore géante censée en imposer avait été désignée par son nom scientifique, poétique et pas du tout terrifiant, mais il était impossible de le changer.

\section{Travailler en groupe ou seule}

Selon les projets, il peut y avoir un dialogue, voire une coopération avec d'autres: traducteurs (même langue ou autre langue), relecteurs, chefs de projet, développeurs, éditeur, responsable LQA?.

XZ: J'aime travailler en groupe. C'est agréable de discuter de problèmes de traduction avec d'autres traducteurs ou des relecteurs. Les développeurs sont très utiles pour les

6. Forme romanisée du mandarin.

7. Linguistic quality assurance ou $Q A$, assurance qualité. 
questions de contexte, mais n'ont souvent pas le temps de répondre à tout avant la date de livraison.

JW: Je travaille souvent seule, en dehors des contacts avec un chef de projet.

SB: Certaines agences gèrent ça très bien, d'autres moins. Ca s'améliore récemment avec de nouvelles plateformes et de meilleurs processus de QA. On reçoit aussi plus de feedback qu'avant, ce qui est très utile! La plupart des chefs de projet sont traducteurs eux-mêmes, donc ils comprennent et cherchent à aider.

EM: J'ai eu deux expériences radicalement opposées. Une où c'était le néant, on ne pouvait pratiquement poser aucune question, l'autre où j'ai vraiment apprécié de pouvoir échanger sur la plateforme Trello avec toi [SM] et les chefs de projet sur nos questions de traduction.

SM: Si l'agence fait bien les choses, c'est très motivant. Sur un projet avec Elisabeth, l'agence avait créé un espace Trello, où tout le monde communiquait, y compris entre langues différentes. Je me souviens que nous avions discuté ensemble du nom français d'un type de créatures de petite taille. Nous avions eu l'information que ces NPC ${ }^{8}$ allaient virer méchants et ça nous a conduites à choisir le suffixe péjoratif-ard plutôt que le sympathique -et ou -ot, neutre.

\section{Traduction, localisation ou adaptation?}

À propos de jeu vidéo, l'emploi du terme "localisation» (stricto sensu, adaptation à un marché spécifique, y compris en remaniant profondément le contenu, voire la mécanique du jeu) est traditionnel, qu'il s'agisse de traduction, avec donc des éléments d'adaptation, ou de vraie localisation pour un pays en particulier.

EM: Une difficulté est le foisonnement du français par rapport au russe: le français utilise des articles, beaucoup plus de prépositions, etc. et on se retrouve avec des segments trop longs, ce qui oblige à adapter, parfois beaucoup.

SM: Certaines adaptations sont linguistiques. J'ai eu un cas où des confréries de personnages avaient des animaux

8. Non-playing character ou PNJ, personnage non joveur. 
emblèmes dangereux ou majestueux (loup, aigle). Problème, l'un d'entre eux était le mouflon, qui malgré ses grandes cornes a un nom français aux consonances tellement "nounours» qu'aucun joueur n'aurait pris la confrérie au sérieux. J'ai dû trouver une solution autre qui convienne quand même aux images. Il faut aussi parfois faire des choix, comme pour cette vieille dame tzigane, "forcément voleuse", dans un jeu russe. J'ai déplacé l'insulte de son appartenance ethnique à son caractère et averti le chef de projet.

XZ: II n'existait pas de système de classification des jeuX en Chine jusqu'au lancement, à l'essai, en décembre 2020, de trois catégories (moins de 8 ans, moins de 12 ans et moins de 16 ans), sans catégorie adulte. Des adaptations culturelles s'imposent donc souvent sur le marché chinois, concernant des questions de protection des enfants, de mots grossiers, de références sexuelles, à la nudité ou aux comportements criminels.

SB: Je suis néo-zélandaise et en localisation, on me dit que je ne suis pas assez anglaise pour le marché britannique et pas assez américaine pour les États-Unis. Ça ne correspond pas du tout à la vie réelle. Le secteur est très conservateur concernant les façons de s'exprimer multiculturelles au sein d'un même pays. Les États-Unis et le Royaume-Uni ont bien plus de variantes de l'anglais que le monde de la localisation ne le dit. On pourrait laisser exister une plus grande polyphonie de locuteurs natifs, comme l'acteur Temuera Morrison qui a apporté son accent néo-zélandais à la série Star Wars.

\section{Le cas particulier de l'anglais}

L'anglais est la langue internationale de l'informatique, d'internet et du gaming. On ne peut l'ignorer dans aucune paire linguistique, même si les problèmes liés à son omniprésence se déclinent différemment.

SM: Pour le français, il y a des arbitrages à faire. D'un côté, les gamers utilisent tous un mélange d'anglais et de français qui, utilisé dans la traduction, peut être un moyen d'apporter crédibilité ou précision, quand il n'y a pas consensus sur le terme français. De l'autre, il faut veiller à ne pas tomber dans le charabia. J'ai toujours ce problème avec le mode de combat skirmish. Faut-il traduire, sachant que le terme "escarmouche» 
n'évoque pas franchement la lutte sans merci de féroces guerriers et que «tout le monde dit skirmish de toute façon»?

SB: Je suis installée en France et je relis beaucoup de projets rédigés en anglais par des gens presque bilingues. Même si leur anglais est excellent et que je comprends leur attachement d'auteur et d'artiste à leur création, ils n'ont pas les réflexes d'un locuteur natif. Je trouve donc souvent du franglais, comme cette créature à l'apparence de mante religieuse pour laquelle le pronom utilisé était le féminin she au lieu du neutre it.

Puisque "tout le monde parle anglais», on peut avoir à négocier avec le client non anglophone natif sur la traduction anglaise de tel ou tel terme, telle ou telle expression. Négociations qu'on perd parfois.

JW: Certains clients veulent absolument éviter les répétitions et ça a pu conduire à des décisions suboptimales à mon avis. Un exemple, pour осколок раковины, ma suggestion était shell fragment ("fragment de coquillage»). Le client a choisi shell shard ("éclat de coquillage») pour éviter de répéter fragment.

\section{Traductrice dans un monde d'hommes}

Environ trois quarts des traducteurs sont en fait des traductrices. Le monde du jeu vidéo, lui, est plutôt masculin ou se voit comme tel, autant côté production que chez les gamers, par défaut gameurs, malgré le nombre significatif de gameuses.

SB: Je rencontre souvent une vision du monde centrée sur les hommes. L'univers du gaming est, bien sûr, très masculin. Quand je travaille sur un texte, j'essaie délibérément de rendre le jeu attractif pour un public et masculin et féminin. En tant que femme, je peux aider à tempérer les aspects les plus machos des dialogues pour donner quelque chose de plus équilibré et de plus crédible. Je pense que c'est bon pour les jeux, qui attireront ainsi une plus large clientèle.

SM: En ce qui me concerne, à part des consœurs, presque toutes les personnes rencontrées professionnellement dans ce milieu étaient des hommes. D'une manière générale, ça s'est toujours bien passé, mais je me souviens que sur un wargame, un développeur à qui je demandais si un pseudonyme 
cité dans un blog faisait référence à un homme ou à une femme, pour savoir comment faire les accords grammaticaux en français, m'a répondu que c'était «un jeu brutal, pas pour les filles». Le chef de projet, un homme lui aussi, était gêné. Il a fait en sorte de m’obtenir la réponse.

Mais, mais...

XZ: J'ai travaillé avec beaucoup de développeuses et de chefs de projet femmes. Je ne vois pas le secteur du gaming comme un monde d'hommes et n'ai pas rencontré de problème pour l'instant.

\section{Et le plaisir dans tout ça?}

\section{As-tu un genre de jeu et/ou un type de contenu favori?}

EM: Les plus agréables à traduire sont les parties narratives: même si elles peuvent demander un peu de recherche documentaire (par exemple pour un jeu historique), on a le plaisir d'écrire un texte cohérent, avec des phrases, qui autorise un minimum de choix stylistique, et ce «tout» (même d'un paragraphe) donne l'impression de maitriser ce qu'on fait.

JW: Les jeux dans des univers fantasy ou d'horreur, les jeux de rôle. Je préfère les parties narratives et les dialogues. C'est le type de contenu sur lequel je travaille le plus souvent, avec I'UI.

SB: Les jeux de rôle fantasy et les jeux de simulation. Les dialogues sont mes contenus préférés. C'est créatif, comme de traduire une pièce de théâtre ou un scénario de film, mais avec des personnages numériques au lieu d'acteurs.

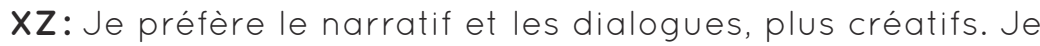
trouve les parties juridiques, comme les contrats de licence d'utilisateur, ennuyeuses.

\section{Qu'est-ce que tu aimes dans la traduction de jeu vidéo?}

$X Z$ : La créativité que la traduction de jeux exige et les univers, à la création desquels je participe dans la langue-cible.

JW: J'aime l'aspect créatif et le fait d'imaginer le joueur s'immergeant dans le contenu que j'ai créé.

SB: Découvrir les histoires et les personnages, participer à un projet créatif et créer quelque chose de cool.

SM: J'aime l'alternance entre le très technique et le très créatif: le défi du nombre limité de caractères dans la partie UI, toute cette terminologie codifiée, les listes d'armes, 
d'objets, etc. mais aussi le travail de transcréation pour les parties narratives et les dialogues, les noms de lieux ou de personnages. Ca me donne la satisfaction de jover de toute la gamme de mes compétences de traduction.

\section{Rêvons un peu}

J'ai demandé aux traductrices et à d'autres acteurs du monde du gaming (Fedor Bonch-Osmolovskiy, responsable d'un studio de localisation, Natalie Mikkelson, auteure de jeu, Ambroise Garel, journaliste jeu vidéo, et Andrew Livy, développeur) de lâcher la bride à leur imagination.

$\mathrm{Si}$, d'un coup de baguette magique, vous pouviez changer un aspect du monde de la traduction de jeu vidéo, que choisiriez-vous?

JW: Plus de transparence et pouvoir se donner le temps de faire les choses bien, cf. les soucis rencontrés par Cyberpunk $2077^{9}$.

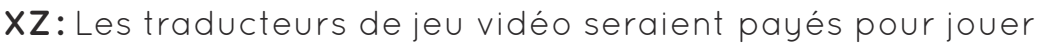
au jeu avant de le traduire.

SB: Ce serait mieux payé. On consacrerait plus de soin et de temps aux étapes de traduction et de relecture.

SM: Les clients finaux se débarrasseraient de leur gestion chaotique ou irréaliste du calendrier et accepteraient que ça prenne le temps que ça doit prendre.

Le studio de localisation: Dans mes rêves, il serait possible d'envoyer un responsable localisation expérimenté dans chaque studio ou chez chaque éditeur qui nous passe commande.

L'auteure de jeu: Dans un monde idéal, il y aurait un moyen facile de vérifier la qualité du travail ou d'avoir accès à des traducteurs validés. Je fais partie d'un petit studio indé, nous n'avons pas de gros département $Q A$. On peut se retrouver à devoir faire confiance les yeux fermés sur toute la durée d'un projet, ce qui est assez angoissant vu les sommes engagées, surtout si un traducteur cesse de répondre ou ne fait pas les vérifications demandées.

9. Jeu du studio CD Projekt Red dont la première version, sortie après des crunchs (périodes de marche forcée pour l'équipe travaillant sur un jeu) et des reports à répétition, était truffée de bugs. 
Le journaliste spécialisé: Si j'avais une baguette magique, je ferais en sorte qu'à l'instar de celui de l'édition qui accepte qu'une traduction paraisse des mois, voire des années après l'œuvre originale, le monde du jeu vidéo se libère de l'impératif de sortie simultanée de versions localisées. Les traducteurs pourraient travailler sur un projet terminé plutôt qu'en perpétuel changement, disposeraient de davantage de temps pour entrer en contact avec les développeurs et, surtout, du temps nécessaire pour jover au jeu qu'ils traduisent dans des conditions similaires à celles du public auquel il est destiné.

Le développeur: Le problème pour un tout petit studio comme le mien est que je n'ai pas de moyen de savoir si les traductions fonctionnent comme je le veux dans mon jeu. Dans l'idéal, il y aurait un processus de QA. Je comprends le japonais et j'ai relevé des erreurs qui ont pu être corrigées, mais pour les autres langues, je n'ai absolument aucune idée de ce qu'il en est.

Dans un monde parfait, à quoi ressemblerait le projet idéal de traduction de jeu?

SB: J'adorerais travailler sur un jeu du début à la fin en étant intégrée à l'équipe du studio. J'aurais assez de temps pour tout peaufiner, pour sculpter chaque phrase avec la même exigence que pour un roman.

JW: Avant de commencer, j'aurais accès à tous les détails de l'histoire et des personnages, aux storyboards, aux choix de création des persos, des paysages, des villes, etc. Sur demande, j'aurais aussi accès à des vidéos montrant le gameplay, comment se jove le jeu. Le délai prendrait en compte le temps de me familiariser avec tout ça, de traduire et de recevoir des réponses aux questions, qui seraient moins nombreuses car j'aurais le contexte nécessaire. J'aimerais aussi des fichiers audio des voix des personnages. Le fichiersource contiendrait une colonne indiquant où dans le jeu et entre quels personnages apparaissent les dialogues. La rémunération suivrait, je serais au générique en tant que traductrice et je recevrais un exemplaire gratuit du jeu.

SM: Une équipe serait constituée, avec des traducteurs, une personne chargée de faire la liaison avec les créateurs du jeu, une autre d'organiser les ressources terminologiques, images, réponses des développeurs, etc., un relecteurcoordinateur pour chaque langue, qui ferait l'alignement 
global et dialoguerait avec les traducteurs. Tout ça avec le temps de faire un vrai travail collectif.

EM: II me paraît indispensable d'avoir des captures d'écran et des explications détaillées sur l'emplacement de chaque string, ou peut-être de pouvoir jouer au jeu directement.

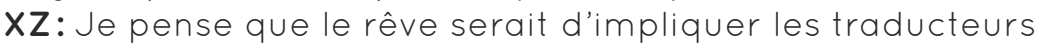
dans tout le processus de développement, depuis le stade des concepts. II y aurait un "traduction thinking» comme il y a un design thinking. Je trouve que les jeux devraient être développés du début à la fin en fonction de la traduction et de la distribution internationale.

Le développeur: La traduction est souvent un processus externe qui intervient après la rédaction du script principal. Dans l'idéal, les traducteurs seraient impliqués dès l'écriture. Le résultat serait bien meilleur dans chaque langue-cible qu'avec «juste» une traduction. Malheureusement, le coût serait prohibitif pour les petits studios.

Le journaliste spécialisé: Le projet de rêve serait une collaboration entre traducteur et petit développeur indépendants, qui permettrait une approche artisanale. Cela donnerait le temps de discuter des enjeux de l'adaptation, de mettre en contact une personne disposant du temps nécessaire pour traduire l'intégralité du jeu et une autre qui, parce qu'elle l'a développé, en a une vision d'ensemble et en saisit pleinement l'intention. On aurait alors des conditions proches d'une relation idéale entre auteur et traducteur.

Le studio de localisation: Ce serait un projet avec différents types de textes, du voiceover et la possibilité de faire la LQA. II y aurait des tonnes de documents de référence et les devs répondraient aux questions sur le contenu. Ce serait compliqué, mais du compliqué gratifiant.

L'auteure de jeu: Toute une équipe superviserait la traduction avec quelqu'un qui créerait un wiki ou un glossaire de jeu que les traducteurs pourraient utiliser facilement, quelqu'un qui gérerait l'organisation technique et les transferts de données, quelqu'un qui répondrait aux questions et ferait la LQA. J'adorerais pouvoir préparer dès le début tous les éléments pour que les traducteurs puissent travailler au mieux.

Tout un programme! 
Un grand merciaux traductrices et à Fedor Bonch-Osmolovskiy du studio de localisation Levsha. Ambroise Garel du magazine Canard PC. Andrew Livy du studio CooCooSqueaky Games et Natalie Mikkelson du studio LoFi Games ${ }^{10}$.

contact@sandramouton.com

Sandra Mouton est traductrice indépendante, de langue maternelle française, travaillant à partir de l'anglais, du russe et du latin à l'intersection du créatif et du technique dans les domaines de la communication, du marketing, de l'art de vivre, des jeux vidéo et du nautisme, ainsi que sur des textes historiques et religieux. Elle est membre de la SFT et Associate de I'Institute of Translation and Interpreting et tweete sous le nom @enFRdansletexte.

10. Contributions de Sasha Barral, Fedor Bonch-Osmolovskiy, Andrew Livy, Natalie Mikkelson, Jessica West et Dr Xiaochun Zhang traduites de l'anglais par Sandra Mouton. 